\title{
Study on Internal Control System Optimization of Chinese Small and Medium-sized Private Enterprises
}

\author{
Cuicui Li \\ Accounting School of Shandong Women's University, Jinan shandong, China \\ sdwulicc@163.com
}

Keywords: Small and Medium-sized Private Enterprises; Internal Control System; Optimization

\begin{abstract}
With the continuous deepening of market-oriented reform, small and medium-sized private enterprises (SMPEs) developed rapidly, gradually becoming the important component of national economy, and exerting more and more important role in national economy. However, in recent years, lots of SMPEs that dominated the market in the last met failure in succession, while the main cause resulting in its operation failure is the failure or deficiency of internal control. At the present stage, SMPEs urgently need to construct and optimize internal control system. Basing on analyzing the development status and internal control status of Chinese SMPEs, this paper proposed suggestion on optimizing internal control system of SMPEs, hoping to promote the sound and steady development of Chinese SMPEs.
\end{abstract}

The rapid development of market economy brought opportunity for SMPEs. SMPEs seized opportunities and developed rapidly, and its proportion in overall national economy increased continuously. At the time of achieving rapid development, the operation system of SMPEs also experienced great change. Lots of enterprises have management problems, making them cannot adapt to severe market competition, even resulting in operation failure. From Giant Interactive Group to Wanjie Group, from Guangdong Aido Electrical Appliances to Sanzhu Oral Liquid, these cases tell us that the deficiency or failure of internal control has become the important bottleneck impeding the development of SMPEs. At the present stage, Chinese SMPEs urgently need to establish and perfect internal control system.

\section{Development Status of SMPEs}

With the continuous development of market economy, SMPEs gradually developed into the important component of national economy. The development of SMPEs generally experienced three stages: capital accumulation, exploration and development, and knowledge management. At the present stage, SMPEs occupy important position in local economy, especially county economy, becoming the entity of investment, tax payment, and offering employment post. Relevant data show that in 2013, China's private economy contributed over $60 \%$ of total GDP.

\section{Internal Control Status of SMPEs}

\subsection{Insufficient Internal Control Consciousness}

It has become an indisputable fact that SMPEs have more problems and big risks. Therefore, SMPEs should promote more rapid and better development via establishing and perfecting internal control system, standardizing enterprise management, preventing operation risk, and improving 
operation efficiency and effect. However, the current situation is that most SMPEs have not established perfect internal control system, and managers of some SMPEs even do not know about internal control. Even though some SMPEs have established internal control system, due to insufficient emphasis of managers, internal control system is not executed completely, becoming a mere formality and cannot truly exert its control role.

\subsection{Undesirable Internal Control Environment}

Internal control environment is the basic element for enterprise internal control system to exert role. Internal control environment of SMPEs is undesirable, mainly exhibited in: firstly, imperfect corporate governance structure, unsound function, ambiguous division of labor, and no effective counterbalance in power, etc; compounded by power overstepping of managers of SMPEs, corporate governance structure cannot exert its stimulation and restriction role, becoming a mere formality. Secondly, unscientific human resources policy, lack of effective performance evaluation mechanism and perfect stimulation mechanism, and nepotism phenomenon is everywhere, resulting in serious talent loss. Thirdly, lack of excellent corporate culture, most SMPEs just emphasize on pursuing economic benefit and avoiding the discussion on cultural construction, making staff lack the sense of belonging and cohesiveness to enterprise, resulting in not strong enterprise comprehensive competitiveness.

\subsection{Thin Risk Consciousness, and Imperfect Risk Evaluation Mechanism}

Risk consciousness of SMPEs is thin, and most SMPEs have not established complete and effective risk evaluation system. Enterprise managers only passively respond to risks only when facing risks, and responding strategy is implemented as per personal experience. Although few SMPEs have established risk evaluation mechanism, risk management tool of some enterprises is improper and some enterprises lack relevant risk management principal, resulting in that risk evaluation mechanism cannot really exert role.

\subsection{Chaos in Control Activity}

Enterprise internal control activity mainly includes responsibility assignment control, authorization approval control, budget control, property protection control, and accounting system control. Currently, most SMPEs have conducted responsibility assignment control and property protection control, and only few enterprises carried out authorization approval control and budget control, etc. It brought great management loophole and risk to enterprises, and seriously impeded effective execution of enterprise internal control system. In addition, as managers pay less attention to internal control and staff quality is not high, etc, it has resulted in that the execution of some SMPEs with perfect setting in internal control activity is greatly reduced. Moreover, important control activity cannot be executed strictly, and internal control activity performs practically no function.

\subsection{Unsmooth Information and Communication}

Information and communication of SMPEs exist problems, mainly exhibited in: there exists basically no problem in upward to downward information communication, while downward to upward information feedback is weak, illustrating that enterprise has no perfect information feedback mechanism; as to external information communication, most SMPEs have less communication and exchange with banks or competent government authorities, thus external information is relatively blocked. In addition, due to the reasons of scale and cost restriction, informatization extent of SMPEs is generally low. It also influenced enterprise information and communication, which is not beneficial to the effective execution of enterprise internal control. 


\subsection{Lack of Effective Internal Supervision Evaluation}

Internal supervision function is mainly achieved via internal audit. Currently, internal audit institution established in part of SMPEs is affiliated to relevant department with poor independence; some SMPEs basically have no internal audit institution, and not carried out any internal audit, the de facto deficiency of internal audit make enterprise lack effective internal supervision. In addition, most SMPEs have not established effective internal supervision evaluation system, resulting in that the execution effect of internal control cannot be fed back. It is more difficult to conduct timely adjustment and improvement on internal control system, which is not beneficial to the achievement of internal control target.

\section{Suggestion on Internal Control System Optimization of SMPEs}

\subsection{Improving Emphasis Extent on Internal Control}

On the one hand, the publicity and promotion strength on internal control should be strengthened, and SMPEs should be conscious of the necessity of establishing and perfecting internal control system via some typical successful and failure cases. On the other hand, the quality of enterprise staff, especially the quality of management should be improved via the methods including training and engaging in advanced studies; and internal control target should be incorporated into annual performance assessment, enabling enterprise staff to participate in the construction of internal control system, actively and initiatively execute and promote the achievement of internal control target.

\subsection{Optimizing Internal Control Environment}

Internal control environment involves various aspects of enterprise, and directly influences the operation effect of enterprise internal control. SMPEs should optimize internal control environment from the following several aspects: firstly, establishing and perfecting corporate governance structure. The independence and effectiveness of decision-making and business discussion of board of directors should be improved via optimizing member structure of board of directors, defining director liability, and punishing severely directors not fulfilling duties. The proportion of independent director should be increased, executive ability of member of board of supervisors should be improved, and the independence of board of supervisors and its role exertion should be guaranteed. The responsibilities and obligations among board of shareholders, board of directors, board of supervisors and the management should be streamlined, and effective internal stimulation and competition should be formed, so as to promote the effective operation of internal control. Secondly, scientific human resource policy should be established. SMPEs should establish and perfect recruitment and selection mechanism, appoint people on their merit, formulate reasonable award and punish system, and thus stimulate work enthusiasm and creativity of staff. In addition, SMPEs should strengthen staff participation, and improve the sense of belonging and responsibility of staff, so as to let them work more hard. Thirdly, enterprise cultural construction should be strengthened. SMPEs should let staff fully participate in enterprise cultural construction via various kinds of publicity and education activity. Especially, managers of SMPEs should exert model leading role and establish positive corporate culture suitable to enterprise characteristics, and create excellent atmosphere of internal control by virtue of invisible influence of corporate culture.

\subsection{Establishing and Perfecting Risk Evaluation Mechanism}

With the rapid development of market economy, market competition becomes increasingly severe, 
thus the capability of SMPEs in evaluating and responding to risk becomes particularly important. Therefore, SMPEs should improve risk prevention consciousness, construct perfect and effective risk evaluation mechanism, reasonably apply risk management tool, establish scientific risk warning system and emergency processing system, and clarify risk management responsibility to people. As to liability ascertaining of major risk management fault, long-term mechanism on risk management should be formed, so as to truly improve risk management ability and efficiency.

\subsection{Perfecting Internal Control Activity, and Executing Practically}

Control activity is the essential link in internal control, SMPEs should combine self characteristics to design internal control activity; standardize responsibility assignment control, authorization approval control, budget control, and accounting system control, establish perfect internal control activity. Meanwhile, staff participation consciousness should be improved via exerting the demonstration role of enterprise managers, so as to form excellent internal control atmosphere and improve executive force of internal control activity. In addition, SMPEs should incorporate the execution effect of internal control into staff performance assessment, promote staff to conscientiously execute internal control activity, and feasibly exert the role of internal control.

\subsection{Constructing Effective Information and Communication Mechanism}

Enterprise information and communication status directly influence the operation efficiency of its internal control. Therefore, SMPEs should establish effective information and communication mechanism. On the one hand, SMPEs should establish smooth communication channel via optimizing organizational structure, formulating formal meeting system and informal suggestion system, and constructing information feedback system, so as to improve the communication status of SMPEs and achieve effective communication. On the other hand, for achieving long-term development, SMPEs must accelerate informatization construction on the basis of considering cost effective principle, engage specialized personnel to establish perfect information system, timely and effectively provide relevant information, and improve the efficiency and effect of enterprise internal control. Moreover, SMPEs should strengthen the communication and exchange with competent government department, tax department, and bank; timely understand relevant policies and positively utilize policy, so as to promote the faster and better development of enterprise.

\subsection{Strengthening Internal Supervision Evaluation}

SMPEs should establish special internal audit institution, allocate internal auditing staff with exquisite business, establish and perfect internal audit rules and regulations, so as to guarantee that internal audit institution and staff can carry out work objectively and independently. In addition, SMPEs should establish perfect internal supervision evaluation mechanism, carry out real-time monitoring on the implementation effect of internal control system, timely feed back monitoring situation, and continuously adjust internal control system as per feedback results, so as to make internal control system be more perfect and effective. Moreover, SMPEs should further strengthen internal supervision evaluation, improve the capability of enterprise in preventing operation risk, and promote the sustainable development of SMPEs via above measures.

\section{Conclusions}

As the important component of national economy, if SMPEs want to achieve sound and long-term development, establishing and perfecting internal control system is an irresistible trend. Therefore, combining with self characteristics and operation environment, SMPEs should optimize internal 
control system via measures including improving the emphasis level on internal control, optimizing internal control environment, establishing and perfecting risk evaluation mechanism, perfecting internal control activity, constructing effective information and communication mechanism, and strengthening internal supervision evaluation, thereby improving its risk prevention capability and comprehensive competitiveness.

\section{Acknowledgement}

This research was financially supported by Shandong Provincial High School Humanities and Social Sciences Research Program (Grant NO. J13WG59) and Shandong Women's University Youth Scientific Research Program (Grant NO. 2012YB05).

\section{References}

[1] Li Yunda, Liu Shebing. Study on Status and Problems of Internal Control of Private Enterprises and Its Perfection Countermeasures. Journal of Jiangsu Teachers University of Technology, 2013(10) .

[2] Chang Dandan. Study on Internal Control of Chinese Small and Medium-sized Enterprises. Master's Dissertation of Shanxi University of Finance and Economics, 2012.

[3] Yang Xiongsheng. Study on Category Definition of Internal Control. Accounting Research, 2011(8).

[4] Zhao Zhiquan, Li Yunda. Humble Opinion on Basic Norm of Enterprise Internal Control. Communication of Finance and Accounting, 2009(6).

[5] Nathaniel M. Stephens. Corporate Governance Quality and Internal Control Reporting under SOX Section302.Accounting Horizons, 2008.

[6] AICPA . Auditing an entity's internal control over financial reporting in conjunction with the financial statement audit reporting on an entity's internal control over financial reporting,2003.

[7] Committee of Sponsoring Organizations of the Treadway Commission(COSO). International Control-Integrated Framework,1992. 